\title{
Panel suggests big changes at Japan's leading university
}

Tokyo. The physics department of Tokyo University, one of Japan's biggest and best research departments, has serious deficiencies in organizational structure and physical facilities despite producing "outstanding" research, according to a committee of distinguished foreign and Japanese scientists that conducted the first external review of a Japanese university department. The committee, in a report released on 30 March, makes a series of recommendations that, if implemented, could have a profound effect on university research throughout Japan.

The review, carried out by the committee during a three-day visit in January (see Nature 361, 196; 1993), is an initiative of Akito Arima, president of Tokyo University, who has pushed hard for reform of Japan's universities. The committee's report was released the day before Arima's retirement and thus its fate lies with his successor, Hiroyuki Yoshikawa. Senior officials at the Ministry of Education, Science and Culture are confident that Yoshikawa will take up Arima's lead.

The physics department, with 35 professors and associate professors in fields including astrophysics, biophysics and nonlinear dynamics and fluid mechanics, is already in the forefront of university reform. The department has modified the traditional koza system, which divides departments into independent subfields ruled by one professor with an associate professor and two research associates under his command. Instead, professors and associate professors now have equal status, and several research associates have been promoted to associate professor.

Nevertheless, the committee members find "much room for improvement". They note that nearly all of the younger (under 40) department members are either research associates, a tenured position with a mixture of teaching and research responsibilities but with little immediate prospect of promotion or the chance to develop a strong independent research programme, or postdoctoral fellows, who take direction from one professor and have little opportunity to pursue their own research.

The committee recommends that the position of research associate should gradually be converted to a non-tenured assistant professor position with greater responsibilities for research and teaching. It also calls for a large increase in the number of postdoctoral fellows.

The committee also notes the absence of women faculty members and foreign faculty members and recommends a "special effort" to recruit women at every level. "This apparent failure to bring exceptionally talented physicists, regardless of their sex or country of national origin, to the Physics Department ... means that students and faculty alike are deprived of the opportunity of having daily contact with some of the leading physicists of our time."

The committee recommends that all new faculty appointments should be "widely advertised outside Japan" without requiring candidates to speak Japanese. It suggests that the relatively poor English of the students should be improved by greater exposure to English-speaking faculty and by attendance at international conferences.

Pointing to physical conditions that are "miserable and utterly unacceptable", the committee says that there is an urgent need to create more space and a better working environment even before new buildings are erected. The committee also comments on the lack of communication between different sections of the department and calls for greater interaction through regular colloquia and informal discussions.

The report says the quality of students is among "the very best" in the world (apart from their lack of ability in English). But it points out that the curriculum for undergraduates is too rigid and does not allow talented students to move at their own pace. The committee members also suggest that graduate students should be given greater freedom to change research topics and supervisor, and that students be allowed to evaluate faculty members (a revolutionary idea in Japan)

Robert Geller, a US geophysicist and one of the few permanent foreign faculty members at Tokyo University, says he agrees with the "spirit" of the committee's recommendations but that converting research associates to assistant professors would create a system of "all chiefs and no indians". He believes that a chronic lack of technicians in Japan's universities must first be addressed, however, because associates now fill the role of technicians.

The marginal English skills of students is not just a function of language, according to Geller. "Students are unable to express themselves even in Japanese", he says, adding that the Japanese education system fails to teach students "how to write clear reports, debate and make speeches".

Akiyoshi Wada, a former member of the physics department and former dean of the faculty of science, also points to the committee's failure to highlight the shortage of technicians. But he says the overall report is "excellent" and that "the committee has achieved more in three days than a Japanese government committee would in one year".

David Swinbanks

\section{Indian scientists decry favouritism of research system}

New Delhi. A majority of Indian scientists believe that people are promoted on the basis of favouritism rather than on the quality of their work and that criteria such as professional contacts and loyalty to a superior are weighted more heavily than the number and impact of papers published, awards received or patents granted.

This is one of the findings of a survey conducted by the National Institute of Science, Technology and Development Studies (NISTADS) examining the disproportionately low impact on global science of the vast numbers of Indian scientists. Some 1,450 scientists at all levels in universities, government research institutions and industries were asked about working conditions, professional norms and other factors relating to the conduct of science.

Most of those polled were dissatisfied with the prevailing ethical and professional standards and were particularly unhappy with the way in which research is evaluated, with the reward system and with recruitment and promotion practices. More than 55 per cent believe that knowing the right people plays an important role in obtaining research grants and that institutions have failed to discourage poor performance and to provide the proper research environment.

The survey found that Indian scientists react badly to criticism, hesitate to criticize their colleagues and seldom communicate or interact either with their immediate colleagues or with scientists outside their own institutes. There is a lack of institutional support for mobility and only the most senior scientists have opportunities to travel.

NISTADS found that India's lacklustre performance stems more from poor management of the research system than from a lack of facilities. The authors of the study, published in the February issue of the Journal of Scientific \& Industrial Research (52, $81-94$; 1993) conclude that existing policies "are neither conducive for nurturing excellence nor for the promotion of applied science".

K. S. Jayaraman

\section{Correction}

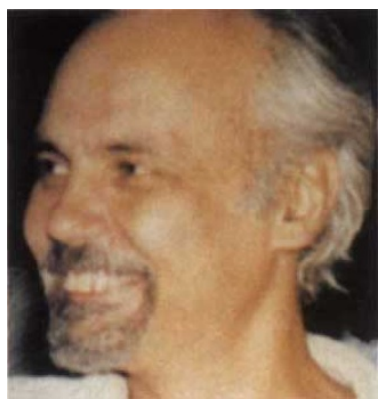

A recent article (Nature $\mathbf{3 6 2}$ 198; 1993) about the appointment of Fotis Kafatos as director of the European Molecular Biology Laboratory was accompanied by the wrong photograph. Kafatos appears left. 